\title{
From Royal Road To Epistatic Road For Variable Length Evolution Algorithm
}

\author{
Michael Defoin Platel ${ }^{1,2}$, Sebastien Verel ${ }^{1}$, Manuel Clergue ${ }^{1}$ and Philippe \\ Collard $^{1}$ \\ 1 Laboratoire I3S, CNRS-Université de Nice Sophia Antipolis \\ 2 ACRI-ST
}

\begin{abstract}
Although there are some real world applications where the use of variable length representation (VLR) in Evolutionary Algorithm is natural and suitable, an academic framework is lacking for such representations. In this work we propose a family of tunable fitness landscapes based on VLR of genotypes. The fitness landscapes we propose possess a tunable degree of both neutrality and epistasis; they are inspired, on the one hand by the Royal Road fitness landscapes, and the other hand by the NK fitness landscapes. So these landscapes offer a scale of continuity from Royal Road functions, with neutrality and no epistasis, to landscapes with a large amount of epistasis and no redundancy. To gain insight into these fitness landscapes, we first use standard tools such as adaptive walks and correlation length. Second, we evaluate the performances of evolutionary algorithms on these landscapes for various values of the neutral and the epistatic parameters; the results allow us to correlate the performances with the expected degrees of neutrality and epistasis.
\end{abstract}

\section{Introduction}

Individuals in Genetic Algorithms (GA) are generally represented with strings of fixed length and each position of the string corresponds to one gene. So, the number of genes is fixed and each of them can take a fixed number of values (often 0 and 1). In variable length representation (VLR), like Messy GA or Genetic Programming, genotypes have a variable number of genes. Here, we consider VLR where a genotype is a sequence of symbols drawn from a finite alphabet and a gene is a given sub-sequence of such symbols. The main difference with fixed length representation is that a gene is identified by its form and not by its absolute position in the genotype.

Some specific obstacles come with the variable length paradigm. One of the most important is the identification of genes. Indeed, during recombination, genes are supposed to be exchanged with others that represent similar features. So the question of the design of suitable crossover operators becomes essential (see for example [1]). Another difficulty due to variable length is the tremendous amount of neutrality of the search space, as noted in [2]. Neutrality appears at different levels. First, a gene may be located at different positions in the 
genotype. Second, some parts of genotype (called introns) do not perform any functions and so do not contribute to fitness. The last specificity is that variable length strings introduce a new dimension in the search space, which have to be carefully explored during evolution to find regions where fitter individuals prevail. The exploration of sizes seems to be difficult to handle and may lead, as in Genetic Programming, to an uncontrolled growth of individuals (a phenomenon called bloat [3]).

One of the major concerns in the GA field is to characterize the difficulty of problems. One way to achieve this is to design problems with parameters controlling the main features of the search space; to run the algorithm; and to exhibit how performances vary according to the parameters. With fixed length representations, some well known families exist, as the Royal Road functions, where inherent neutrality is controlled by the block size, or the NK-landscapes, where the tunable parameter $K$ controls the ruggedness of the search space. With VLR, there are only a few attempts to design such academic frameworks 4 . Note, for example, the Royal Tree [5] and the Royal Road for Linear GP [1].

\section{Royal Road for variable length representation}

In GA, Royal Road landscapes (RR) were originally designed to describe how building blocks are combined to produce fitter and fitter solutions and to investigate how the schemata evolution actually takes place [6]. Little work is related to RR in variable length EA; e.g. the Royal Tree Problem [5] which is an attempt to develop a benchmark for Tree-based Genetic Programming and which has been used in Clergue et al. [7] to study problem difficulty. To the best of our knowledge, there was no such work with linear structures.

In a previous work, we have proposed a new kind of fitness landscape [1], called Royal Road landscapes for variable length EA (VLR Royal Road). Our aim was to study the behavior of a crossover operator during evolution. To achieve this goal, we needed experiments able to highlight the destructive (or constructive) effects of crossover on building blocks.

To define VLR Royal Road, we have chosen a family of optimal genotypes and have broken them into a set of small building blocks. Formally, the set of optima is:

$$
\left\{g \in G_{\Sigma} \mid \forall l \in \Sigma, B_{b}(g, l)=1\right\}
$$

with

$$
B_{b}(g, l)=\left\{\begin{array}{l}
1 \text { if } \exists i \in[0, \lambda-b] \mid, \forall j \in[0, b-1], g_{i+j}=l, \\
0 \text { otherwise }
\end{array}\right.
$$

and

$-b \geq 1$ the size of blocks

$-\Sigma$ an alphabet of size $N$ that defines the set of all possible letters $l$ per locus

$-G_{\Sigma}$ the finite set of all genotypes of size $\lambda \leq \lambda_{\max }{ }^{3}$ defined over $\Sigma$

\footnotetext{
${ }^{3} \lambda_{\max }$ have to be greater than $\mathrm{Nb}$
} 
- $g$ a genotype of size $\lambda \leq \lambda_{\max }$

$-g_{k}$ the $k^{t h}$ locus of $g$.

The following genotype $g \in G_{\Sigma}$ is an example of optimum, with $\Sigma=\{A, T, G, C\}$ and $b=3$ :

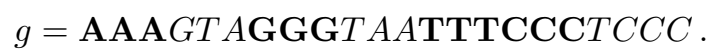

$B_{b}(g, l)$ acts as a predicate accounting for the presence (or the absence) of a contiguous sequence of a single letter (i.e. a block). Note that only the presence of a block is taken into account, neither its position nor its repetition. The number of blocks corresponds to the number of letters $l \in \Sigma$ for which $B_{b}(g, l)$ is equal to one. In the previous example, only boldfaced sequences contribute to fitness ${ }^{4}$. The contribution of each block is fixed and so, the fitness $f_{N b}(g)$ of genotype $g \in G_{\Sigma}$ having $n$ blocks is simply:

$$
f_{N b}(g)=\frac{1}{N} \sum_{i=1}^{N} B_{b}\left(g, l_{i}\right)=\frac{n}{N}
$$

To efficiently reach an optimum, the EA system has to create and combine blocks without breaking existing structures. These landscapes were designed in such a way that fitness degradation due to crossover may occur only when recombination sites are chosen inside blocks, and never in case of blocks translocations or concatenations. In other words, there is no inter blocks epistasis.

\section{$3 \quad$ NK-Landscapes}

Kauffman 8 designed a family of problems, the NK-landscapes, to explore how epistasis is linked to the 'ruggedness' of search spaces. Here, epistasis corresponds to the degree of interaction between genes, and ruggedness is related to local optima, their number and especially their density. In NK-landscapes, epistasis can be tuned by a single parameter. Hereafter, we give a more formal definition of NK-landscapes followed by a summary review of their properties.

\subsection{Definition}

The fitness function of a NK-landscape is a function $f_{N K}:\{0,1\}^{N} \rightarrow[0,1)$ defined on binary strings with $N$ loci. Each locus $i$ represents a gene with two possible alleles, 0 or 1 . An 'atom' with fixed epistasis level is represented by a fitness components $f_{i}:\{0,1\}^{K+1} \rightarrow[0,1)$ associated to each locus $i$. It depends on the allele at locus $i$ and also on the alleles at $K$ other epistatic loci ( $K$ must fall between 0 and $N-1)$. The fitness $f_{N K}(x)$ of $x \in\{0,1\}^{N}$ is the average of the values of the $N$ fitness components $f_{i}$ :

\footnotetext{
${ }^{4}$ Although the last sequence of 'CCC' is a valid block, it does not contribute to fitness since it is only another occurrence.
} 


$$
f_{N K}(x)=\frac{1}{N} \sum_{i=1}^{N} f_{i}\left(x_{i} ; x_{i_{1}}, \ldots, x_{i_{K}}\right)
$$

where $\left\{i_{1}, \ldots, i_{K}\right\} \subset\{1, \ldots, i-1, i+1, \ldots, N\}$. Many ways have been proposed to choose the $K$ other loci from $N$ loci in the genotype. Two possibilities are mainly used: adjacent and random neighborhoods. With an adjacent neighborhood, the $K$ genes nearest to the locus $i$ are chosen (the genotype is taken to have periodic boundaries). With a random neighborhood, the $K$ genes are chosen randomly on the genotype. Each fitness component $f_{i}$ is specified by extension, ie a number $y_{i,\left(x_{i} ; x_{i_{1}}, \ldots, x_{i_{K}}\right)}$ from $[0,1)$ is associated with each element $\left(x_{i} ; x_{i_{1}}, \ldots, x_{i_{K}}\right)$ from $\{0,1\}^{K+1}$. Those numbers are uniformly distributed in the interval $[0,1)$.

\subsection{Properties}

The NK-landscapes have been used to study links between epistasis and local optima. The definition of local optimum is relative to a distance metric or to a neighborhood choice. Here we consider that two strings of length $N$ are neighbors if their Hamming distance is exactly one. A string is a local optimum if it is fitter than its neighbors.

The properties of NK-landscapes are given hereafter in term of local optima: their distribution of fitness, their number and their mutual distance. These results can be found in Kauffman[8, Weinberger[9, Fontana et al. [10].

- For $K=0$ the fitness function becomes the classical additive multi-locus model, for which

- There is single and attractive global optimum.

- There always exists a fitter neighbor (except for global optimum).

- Therefore the global optimum could be reach on average in $N / 2$ adaptive steps.

- For $K=N-1$, the fitness function is equivalent to a random assignment of fitnesses over the genotypic space, and so:

- The probability that a genotype is a local optimum is $\frac{1}{N+1}$.

- The expected number of local optima is $\frac{2^{N}}{N+1}$.

- The average distance between local optima is approximately $2 \ln (N-1)$

- For $K$ small, the highest local optima share many of their alleles in common.

- For $K$ large:

- The fitnesses of local optima are distributed with an asymptotically normal distribution with mean $m$ and variance $s$ approximately:

$$
m=\mu+\sigma \sqrt{\frac{2 \ln (K+1)}{K+1}}, s=\frac{(K+1) \sigma^{2}}{N(K+1+2(K+2) \ln (K+1))}
$$

where $\mu$ is the expected value of $f_{i}$ and $\sigma^{2}$ its variance. In the case of the uniform distribution, $\mu=1 / 2$ and $\sigma=\sqrt{1 / 12}$. 
- The average distance between local optima is approximately $\frac{N \log _{2}(K+1)}{2(K+1)}$.

- The autocorrelation function $\rho(s)$ and the correlation length $\tau$ are:

$$
\rho(s)=\left(1-\frac{K+1}{N}\right)^{s}, \tau=\frac{-1}{\ln \left(1-\frac{K+1}{N}\right)}
$$

\section{Epistatic Road for variable length representation}

In this section, we define a problem with tunable difficulty for variable length EA, called Epistatic Road functions (ER). To do so, we propose to use the relation between epistasis and difficulty.

\subsection{Definition}

Individuals in a variable length representation may be viewed as sets of interacting genes. So, in order to model such a variable length search space, we have to first identify genes and second explicitly define their relations. This can be easily done by extending the VLR Royal Road thanks to dependencies between the fitness contributions of blocks. Thus, genes are designated as blocks and the contribution of a gene depends on the presence of others, exactly as in NKlandscapes.

More formally, the fitness function of an ER-landscape is a function $f_{N K b}$ : $G_{\Sigma} \rightarrow[0,1)$ defined on variable length genotypes. The fitness components $f_{i}$ are defined in 3.1, and the fitness $f_{N K b}(g)$ of genotype $g \in G_{\Sigma}$ is the average of $N$ fitness components $f_{i}$ :

$$
f_{N K b}(g)=\frac{1}{N} \sum_{i=1}^{N} f_{i}\left(B_{b}\left(g, l_{i}\right) ; B_{b}\left(g, l_{i_{1}}\right), \ldots, B_{b}\left(g, l_{i_{K}}\right)\right)
$$

In practice, we use an implementation of NK-landscape with random neighborhood to compute $f_{i}$. We have to ensure that the set of all genotypes having $N$ blocks corresponds to the end of the Road. For that purpose, first we exhaustively explore the space $\{0,1\}^{N}$ to find the optimum value of the NK, then we permute this space in such a way that the optimum becomes $1^{N}$.

\subsection{Tunability}

The properties of an ER-landscape depends on the three parameters $N, K$ and $b$. Although these parameters are not entirely independent, each allows us to control a particular aspect of the landscape. Increasing the parameter $N$ causes the size of both the search space and the neighborhood of genotype to increase. Moreover, as $N$ determines the number of genes to find, the computational effort required to reach the optimum will be more important when high values of $N$ are used. The parameter $b$ controls the degree of neutrality. As $b$ increases the 
size of iso-fitness sets increases. Finally, the parameter $K$ allows to control the number of epistatic links between genes and so the number of local optima. For $K=0$, an ER-landscape will be very closed to the corresponding VLR Royal Road since insertion of a new block in a genotype always increases the fitness. In contrast, for $K=N-1$, with a high level of epistasis, the vast majority of the roads leads to local optima where the insertion of a new block in a genotype always decreases the fitness.

\section{$5 \quad$ Fitness landscape analysis}

Many measures have been developed to describe fitness landscapes in terms of "difficulty". Here, "difficulty" refers to the ability of a local heuristic to reach the optimum. In this section some of those metrics are applied to the ER-landscapes. In particular, we show how difficulty changes according to the three parameters $N, K$ and $b$. The neighborhood of variable length genotypes is different from the neighborhood of fixed length genotypes. To define a neighborhood in ERlandscapes, we use String Edit Distance, like Levenshtein distance [11] which has been already used in GP to compute or control diversity [12, or to study the influence of genetic operators [13]. By definition, the Edit Distance between two genotypes corresponds to the minimal number of elementary operations (deletion, insertion and substitution) required to change one genotype into the other. So two strings in the search space are neighbors if the Edit Distance between them is equal to 1 . Thus a string of length $\lambda$ has $(2 \lambda+1) N$ neighbors.

In order to minimize the influence of the random creation of an NK-landscape, we take the average of the following measures over 10 different landscapes for each couple of parameters $N$ and $K$. We have perform experiments for $N=8$, 10 and 16, for $K$ between 0 and $N-1$ and for $b$ between 1 and 5 .

\subsection{Random walks, autocorrelation function and correlation length}

Weinberger 9,14 defined autocorrelation function and correlation length of random walks to measure the epistasis of fitness landscapes.

A random walk $\left\{g_{t}, g_{t+1}, \ldots\right\}$ is a series where $g_{t}$ is the initial genotype and $g_{i+1}$ is a randomly selected neighbor of $g_{i}$. Then the autocorrelation function $\rho$ of a fitness function $f$ is the autocorrelation function of the time series $\left\{f\left(g_{t}\right), f\left(g_{t+1}\right), \ldots\right\}$ :

$$
\rho(s)=\frac{\left\langle f\left(g_{t}\right) f\left(g_{t+s}\right)\right\rangle_{t}-\langle f\rangle^{2}}{\operatorname{var}(f)}
$$

The correlation length $\tau$ measures how the correlation function decreases and so how rugged the landscape is. More rugged the landscape the shorter the correlation length.

$$
\tau=-\frac{1}{\ln (\rho(1))}
$$

Empirical measures on ER landscapes were performed on $20.10^{3}$ random walks of length 35 for each triplet of parameters $N, K, b$ and for each of 10 
instances of NK-landscapes. The initial genotypes were generated by randomly choosing its length between 0 and $\lambda_{\max }$ and then randomly choosing each letter of the genotype. For those random walks, $\lambda_{\max }$ is equal to $2 \mathrm{Nb}$. For small

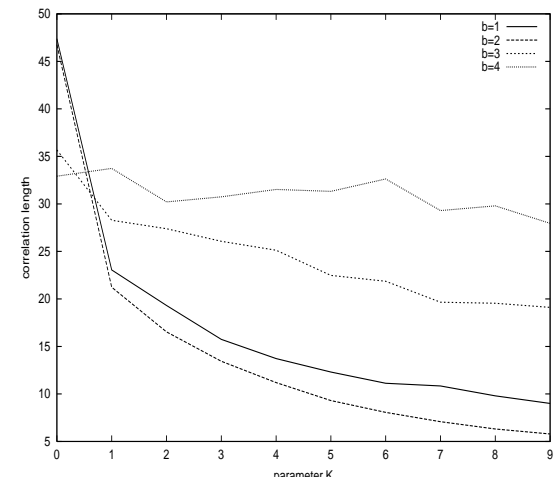

Fig. 1. Mean correlation length of ER-landscapes for $N=10$

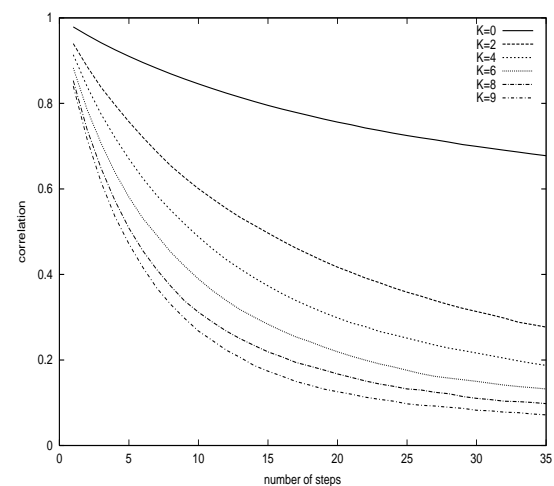

Fig. 3. Autocorrelation function of ER-landscape for $N=10$

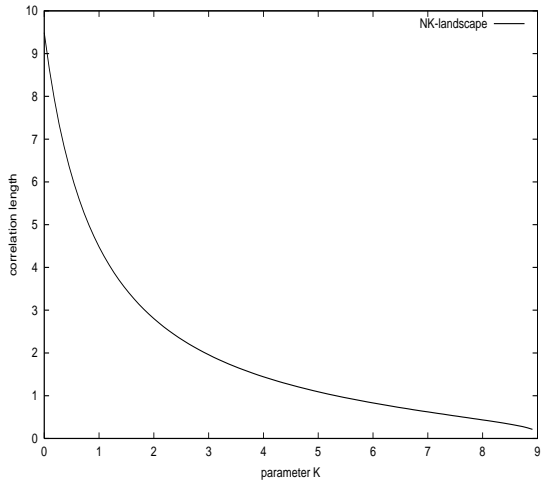

Fig. 2. Theoretical correlation length of NK-landscapes for $N=10$

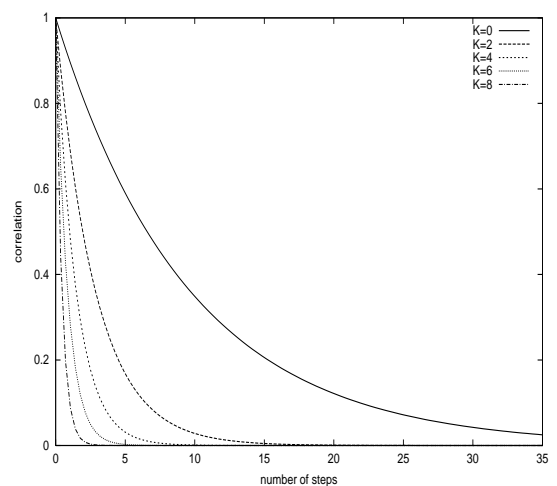

Fig. 4. Theoretical autocorrelation function of NK-landscape for $N=10$

values of $b$, the correlation length decreases quickly (when the parameter $K$ increases, see fig. 1 and 3). As expected, the correlation of fitness between genotypes decreases with the modality due to the parameter $K$. We can compare this variation with the theoretical correlation length of NK-landscapes, given in 3.2 
(see fig. 2 and (4). As $b$ increases, the influence of $K$ on the correlation length decreases. Neutrality keeps a high level of correlation in spite of the increase in modality.

\subsection{Adaptive walks and local optima}

Several variants of adaptive walk (often called myopic or greedy adaptive walk) exists. Here we use the series $\left\{g_{t}, g_{t+1}, \ldots, g_{t+l}\right\}$ where $g_{t}$ is the initial genotype and $g_{i+1}$ is one of the fittest neighbor of $g_{i}$. The walk stops on $g_{t+l}$ which is a local optimum. By computing several adaptive walks, we can estimate:

- The fitness distribution of local optima by the distribution of the final fitnesses $f\left(g_{t+l}\right)$.

- The distance between local optima which is approximately twice the mean of the length $l$ of those adaptive walks.

Empirical measurements on ER landscapes were performed on $2.10^{3}$ random walks for each triplet of parameters $N, K, b$ and for each of 10 instances of NKlandscapes. We used the same initialization procedure as the random walk. The parameter $\lambda_{\max }$ is set to 50 . The distribution of local optima fitnesses is close to

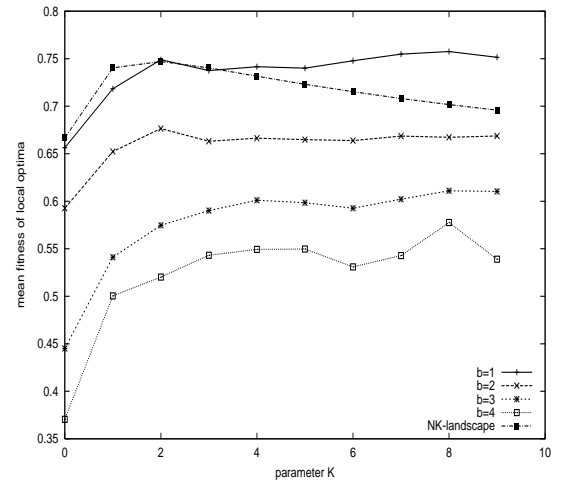

Fig. 5. Mean fitness of local optima of ER-landscapes obtained with adaptive walks for $N=10$

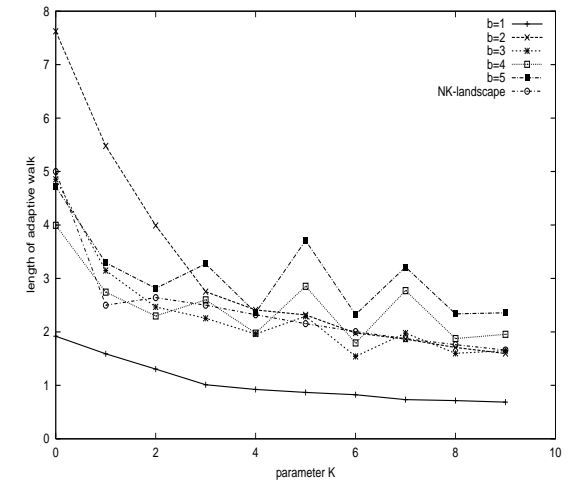

Fig. 6. Mean length of adaptive walks on ER-landscape for $N=10$

normal distribution. The mean fitness of local optima is represented for $N=10$ on Figure ; it decreases with $b$. The variations of the fitness of local optima are great for small values of $K$ but become almost insignificant for medium and high values of $K$. In Figure 6, the mean length of adaptive walks is represented for $N=10$. As expected, it decreases with $K$ for small values of $b$. So, the parameter $K$ increases the ruggedness of the ER-landscape. On the other hand, when $b$ is 
higher, $K$ has less influence on the length of the walk. Indeed, the adaptive walk breaks off more often on neutral plateaux.

\subsection{Neutrality}

A random walk is used to measure the neutrality of ER-landscapes. At each step, the number of neighbors with lower, equal and higher fitness is counted. We perform $2.10^{3}$ random walks of length 20 for each triplet of parameter $N$, $K$ and $b$. The Table 1 gives the proportions of such neighbors for $N=8, K=4$ (they depend slightly on $N$ and $K$ ) and for several values of $b$. The number of equally fit neighbors is always high and is maximum for $b=4$. So, neutral moves are a very important feature of ER-landscapes.

Table 1. Proportion of Lower, Equal and Higher neighbor

\begin{tabular}{|c|c|c|c|}
\hline & \multicolumn{3}{|c|}{$N=8, K=4$} \\
\cline { 2 - 4 } Block size & Lower & Equal & Higher \\
\hline \hline$b=2$ & 7.2 & 85.8 & 7.0 \\
$b=3$ & 2.8 & 94.4 & 2.8 \\
$b=4$ & 0.5 & 98.9 & 0.6 \\
\hline
\end{tabular}

\section{EA performances}

In this section, we want to compare the performances of an evolutionary system on ER-landscapes for various settings of the three parameters $N, K$ and $b$. The performances are measured by the success rate and the mean number of blocks found. In order to minimize the influence of the random creation of NK-landscapes, we take the average of these two measures over 10 different landscapes. 35 independent runs are performed with mutation and crossover rates of respectively 0.9 and 0.3 (as found in $\mathbb{1}$ ). The standard one point crossover, which blindly swaps sub-sequences of parents, was used. Let us notice that a mutation rate of 0.9 means that each program involved in reproduction has a 0.9 probability to undergo one insertion, one deletion and one substitution. Populations of 1000 individuals were randomly created according to a maximum creation size of 50 . The evolution, with elitism, maximum program size of $100\left(\lambda_{\max }\right)$, 4-tournament selection, and steady-state replacement, took place during 400 generations.

\subsection{Results}

We have performed experiments for $N=8,10$ and 16, for $K$ between 0 and $N / 2$ and for $b$ between 2 and 5 . We note that the case $b=1$ is not relevant because the 
optimum is always found at the first generations for all values of $K$. In Figure 8 , we have reported the success rate (over $35 \times 10$ runs) as a function of $K$ for $N=8$. As expected, we see that for $K=0$, the problem is easy to solve for all values of $b$. Moreover, increasing $K$ decreases the success rate and this phenomenon is amplified when high values of $b$ are used. For $N=10$ and 16 , too few runs find the optimum and so the variations of the success rate are not significant. The Figure 7 gives the evolution of the average number of blocks of the best individual found for $N=10, b=4$ and $K$ between 0 and 5 . At the beginning of the runs, the number of blocks found increases quickly then halts after several generations. The higher is $K$, the sooner ends evolution. This behavior looks like premature converge and confirms experimentally that the number of local optima increases with $K$. We have also plotted the average number of blocks of the best individual found as a function of $K$ for $N=16$ (see Fig. 9). We see that this number decreases as $K$ or $b$ increases. These two parameters undoubtedly modify the performances and can be used independently to increase problem difficulty.

In [15], random and adaptive walks have been used to measure problem difficulty in GP. The author has shown that only the adaptive walk gives significant results on classical GP benchmarks. We have computed the correlation between these two measures and the average number of blocks found on ER, for all settings of $N, K$ and $b$. We note that the correlation is 0.71 between the length of the adaptive walk and the number of blocks. Conversely, the length of the random walk seems to be completely uncorrelated to performance.

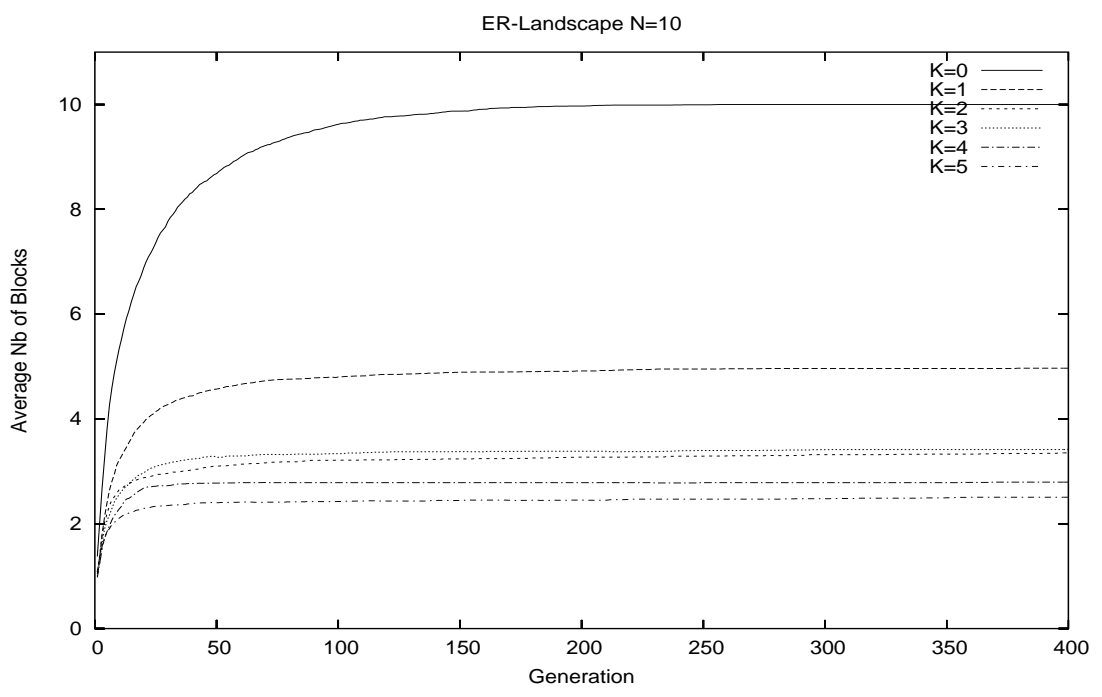

Fig. 7. Evolution of average number of blocks found on ER $N=10$ and $b=4$. 


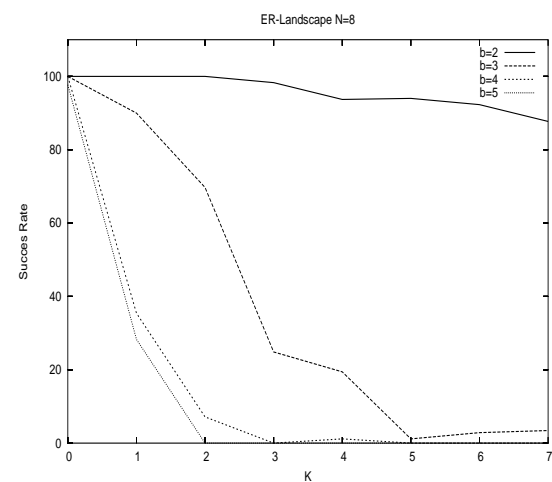

Fig. 8. Success rate as a function of $\mathrm{K}$ on $\mathrm{ER} N=8$.

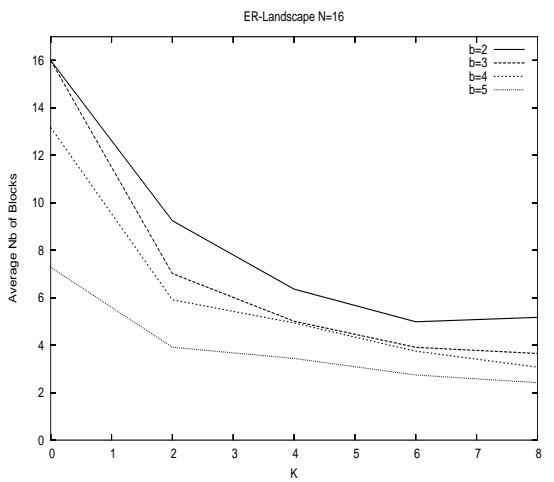

Fig. 9. Average number of blocks found as a function of $\mathrm{K}$ on $\mathrm{ER}$ $N=16$.

\section{Conclusion}

We think that a better understanding of the implications of variable length representations on Evolutionary Algorithms would allow researchers to use these structures more efficiently. In this paper, our goal is to investigate which kind of property could influence the difficulty of such problems. We have chosen two features of search spaces, the neutrality and the ruggedness. So, we have designed a family of problems, the Epistatic Road landscapes, where those features can be tuned independently.

Statistical measures computed on ER-landscapes have shown that, similarly to NK-landscapes, tuning the epistatic coupling parameter $K$ increases ruggedness. Moreover, as for Royal Roads functions, tuning the size block parameter $b$ increases neutrality.

The experiments that we have performed with a VLR evolutionary algorithm, have demonstrated the expected difficulty according to parameters $b$ and $K$. Although our results can not be directly transposed to real world problems, mainly because our initial hypotheses are too simple, in particular about the nature of building blocks, we have a ready-to-use VLR problem of tunable difficulty, which allows us to study the effects of genetic operators and the dynamics of the evolutionary process.

\section{References}

1. Defoin-Platel, M., Clergue, M., Collard, P.: Maximum homologous crossover for linear genetic programming. In: Genetic Programming, Proceedings of EuroGP'2003. Volume 2610 of LNCS., Essex, UK, Springer-Verlag (2003) 194-203 
2. Banzhaf, W., Frankone, F.D., Nordin, P.: Some emergent properties of variable size EAs. Position paper at the Workshop on Evolutionary Computation with Variable Size Representation at ICGA-97 (1997)

3. Langdon, W.B., Poli, R.: Fitness causes bloat. In Chawdhry, P.K., Roy, R., Pan, R.K., eds.: Second On-line World Conference on Soft Computing in Engineering Design and Manufacturing, Springer-Verlag London (1997) 13-22

4. Daida, J.M., Polito, J.A., Stanhope, S.A., Bertram, R.R., Khoo, J.C., Chaudhary, S.A.: What makes a problem GP-hard? analysis of a tunably difficult problem in genetic programming. In Banzhaf, W., Daida, J., Eiben, A.E., Garzon, M.H., Honavar, V., Jakiela, M., Smith, R.E., eds.: Proceedings of the Genetic and Evolutionary Computation Conference. Volume 2., Orlando, Florida, USA, Morgan Kaufmann (1999) 982-989

5. Punch, W.F., Zongker, D., Goodman, E.D.: The royal tree problem, a benchmark for single and multiple population genetic programming. In: Advances in Genetic Programming 2. MIT Press, Cambridge, MA, USA (1996) 299-316

6. Forrest, S., Mitchell, M.: Relative building-block fitness and the building-block hypothesis. In: Foundation of Genetic Algorithms 2. Morgan Kaufman (1993) 109-126

7. Clergue, M., Collard, P., Tomassini, M., Vanneschi, L.: Fitness distance correlation and problem difficulty for genetic programming. In: GECCO 2002: Proceedings of the Genetic and Evolutionary Computation Conference, New York, Morgan Kaufmann Publishers (2002) 724-732

8. Kauffman, S.A.: "The origins of order". Self-organization and selection in evolution. Oxford University Press, New-York (1993)

9. Weinberger, E.D.: Local properties of kauffman's N-k model, a tuneably rugged energy landscape. Physical Review A 44 (1991) 6399-6413

10. Fontana, W., Stadler, P.F., Bornberg-Bauer, E.G., Griesmacher, T., Hofacker, I.L., Tacker, M., Tarazona, P., Weinberger, E.D., Schuster, P.: RNA folding and combinatory landscapes. Physical review E 47 (1993) 2083-2099

11. Levenshtein., V.I.: Binary codes capable of correcting deletions, insertions, and reversals. Soviet Physics-Doklady (1966)

12. Brameier, M., Bhanzhaf, W.: Explicit control of diversity and effective variation distance in linear genetic programming (2001)

13. O'Reilly, U.: Using a distance metric on genetic programs to understand genetic operators (1997)

14. Weinberger, E.D.: Correlated and uncorrelated fitness landscapes and how to tell the difference. Biological Cybernetics 63 (1990) 325-336

15. Kinnear, Jr., K.E.: Fitness landscapes and difficulty in genetic programming. In: Proceedings of the 1994 IEEE World Conference on Computational Intelligence. Volume 1., Orlando, Florida, USA, IEEE Press (1994) 142-147 